\title{
Uncertainty-Aware Guided Volume Segmentation
}

\author{
Jörg-Stefan Praßni, Timo Ropinski, and Klaus Hinrichs
}

\begin{abstract}
Although direct volume rendering is established as a powerful tool for the visualization of volumetric data, efficient and reliable feature detection is still an open topic. Usually, a tradeoff between fast but imprecise classification schemes and accurate but time-consuming segmentation techniques has to be made. Furthermore, the issue of uncertainty introduced with the feature detection process is completely neglected by the majority of existing approaches. In this paper we propose a guided probabilistic volume segmentation approach that focuses on the minimization of uncertainty. In an iterative process, our system continuously assesses uncertainty of a random walker-based segmentation in order to detect regions with high ambiguity, to which the user's attention is directed to support the correction of potential misclassifications. This reduces the risk of critical segmentation errors and ensures that information about the segmentation's reliability is conveyed to the user in a dependable way. In order to improve the efficiency of the segmentation process, our technique does not only take into account the volume data to be segmented, but also enables the user to incorporate classification information. An interactive workflow has been achieved by implementing the presented system on the GPU using the OpenCL API. Our results obtained for several medical data sets of different modalities, including brain MRI and abdominal CT, demonstrate the reliability and efficiency of our approach.
\end{abstract}

Index Terms-Volume segmentation, uncertainty, classification, random walker.

\section{INTRODUCTION}

Although direct volume rendering (DVR) has proven to be a powerful technique for the exploration of volumetric data, its wide-spread practical use has been hampered by mainly two issues. First, the design of effective transfer functions that map data values to optical properties, also called classification, is mostly still a cumbersome and nonintuitive task. Due to the complex relation between transfer function and rendering, a trial-and-error process is often required. Despite more than a decade of intensive research in the field of volume classification, that has yielded many promising transfer function approaches, there still does not seem to be a final solution for this essential task in sight. Volume segmentation techniques, which operate in the spatial domain by classifying voxels directly instead of applying an intermediate transfer function to their data values, could in principle replace the classification step. However, they are usually either too time-consuming for routine use in that they require a massive amount of user interaction, e. g., iterating over all slices and marking the desired structure with high precision, or do not yield sufficiently accurate results for volumes with low signal-to-noise ratio. While sophisticated model-based segmentation approaches exist, they are tailored towards a specific structure and cannot be used for other purposes or when a certain degree of abnormality is exceeded, which may apply to pathological medical cases, such as tumors or ruptures.

The second severe problem of DVR is the lack of reliability. In contrast to traditional slice-wise examination of volume data sets, DVR inherently suffers from occlusion and therefore requires masking through classification and/or segmentation in order to get an occlusionfree view to the structures of interest. This, however, poses the risk of unintentionally masking parts of the structure of interest or erroneously assigning parts of the background to the feature of interest. Both of these effects might lead to critical misinterpretations during visual inspection and to an erroneous quantitative analysis, e. g., when measuring a tumor's volume. Given the well-accepted importance of uncertainty visualization as one of the top visualization research challenges [18], surprisingly few research papers have actually tackled the issue of uncertainty arising through the volume classification process.

- Jörg-Stefan Praßni, Timo Ropinski, and Klaus Hinrichs are with the Visualization and Computer Graphics Research Group (VisCG), University of Münster, E-mail: $\{j$-s.prassni|ropinski|khh\}@math.uni-muenster.de.

Manuscript received 31 March 2010; accepted 1 August 2010; posted online 24 October 2010; mailed on 16 October 2010.

For information on obtaining reprints of this article, please send emailto:tvcg@computer.org.
In this paper we propose a guided segmentation approach, which focuses on uncertainty and is conducted by an interplay between the user and the system. The underlying workflow has been designed as a tight interplay, where each subtask is delegated to the optimal interaction partner. While the human user is rather strong in visual pattern recognition and can reliably estimate an appropriately visualized situation, the strength of the system is vast processing power, which can be exploited to support expensive computations as well as a rough analysis. As shown in Figure 1, in the first step of the workflow, we exploit the probabilistic random walker segmentation algorithm [11] in combination with the high processing power of the GPU to generate segmentations with known uncertainty. In the second step, we analyze this uncertainty information. In order to allow the user to judge and modify the uncertain regions, our system directs the user's attention to regions with high uncertainty in step 3 . Finally, within step 4 the user can inspect the results and refine the starting parameters of the previous segmentation, and the workflow starts over again through the feedback loop. This workflow ensures, that the user is always aware of the currently involved uncertainty, which is essential to judge the reliability of the results.

The main contribution of this paper is an uncertainty-guided volume segmentation workflow, which is based on a GPU implementation of the random walker segmentation algorithm. The user is able to generate a segmentation through the interactive workflow, within which uncertain regions are automatically extracted and presented to the user in the order of their importance. Thus, the user can quickly classify unambiguous regions, which usually constitute the largest part of a data set. The system then directs the user's attention to the uncertain parts of the segmentation and allows the user to locally refine the segmentation, and it ensures that the uncertainty information is conveyed in a reliable way. To our knowledge, the presented system is the first which integrates direct visual feedback of segmentation uncertainty into an interactive segmentation approach.

In the following, we use the term classification synonymously for segmentation, and we do not explicitly refer to transfer function design but we understand it more generally as the assignment of voxels to clusters.

\section{Related Work}

Since volume segmentation is a large area of research, we limit our review to the fields most relevant to our work: supervised volume segmentation as well as volume segmentation systems. Additionally, we cover approaches for visualization of uncertainty in volume data.

Supervised volume segmentation. Based on the supported user interaction, supervised volume segmentation techniques can be cate- 
Feedback Loop

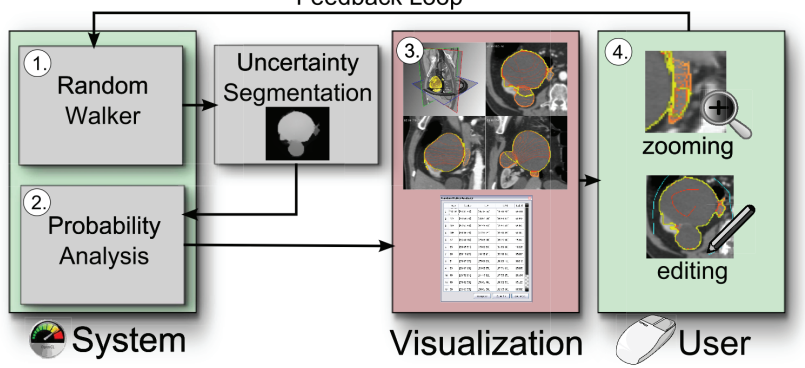

Fig. 1. Proposed workflow: The system generates a random walker solution using seeds that have been defined in previous iterations, analyzes the probabilistic field in order to detect uncertain regions, conveys this uncertainty information to the user by using a table widget as well as integrating it into the 2D and $3 \mathrm{D}$ views, thus allowing the user to select and modify uncertain regions.

gorized into two types [26]: Edge-based methods aim at detecting the boundary between the desired structures and the background. They either take a small set of boundary voxels as user input, or require the user to provide a nearby complete boundary. Region-based techniques consider the region to be extracted as a continuous set of similar voxels, and they expect the user to specify an initial set of seed voxels belonging to the desired object.

The most prominent group of boundary-based segmentation approaches are active contours and level sets [34]. All these techniques require that the user places a contour near the desired boundary, which is then evolved to a local energy minimum. The algorithms mainly vary in the design of the energy functional to be minimized. The complex setup and the limited user interaction have traditionally been considered as severe drawbacks of the level set technique hampering its application in interactive workflows. However, recent presentations of interactive, level set based segmentation tools by Ben-Zadok et al. [2] and Cremers et al. [8] might indicate a change in that respect. The intelligent scissors algorithm [25] is a boundary-based segmentation technique for $2 \mathrm{D}$ images. It computes a minimum-cost path between user-specified boundary points via Dijkstra's shortest-path algorithm. Hastreiter and Ertl [15] have extended it to volume segmentation by considering inter-slice relations and a 3D filter to compute the local cost.

Region growing is a basic region-based segmentation method that starts from an initial set of seed points and iteratively adds neighboring voxels when they satisfy a similarity criterion, which is usually based on intensity thresholds [16] or a diffusion metric [36]. Although in many cases allowing fast segmentation results that correspond well to the perceived boundaries, the choice of the homogeneity criterion becomes difficult for objects with weak boundaries [40]. The graph cuts technique proposed by Boykov and Jolly [4] interprets the image as a graph, weighted to reflect intensity changes. While the algorithm can be easily generalized to 3D, it suffers from the "small cut" problem: since the algorithm returns the smallest cut separating the seeds, a small number of seeds might not be adequate. Grady [11] introduced the random walker technique as a probabilistic, seeded image segmentation approach. Like graph cuts, it views the volume as a weighted graph, whose nodes correspond to the voxels. The basic idea is to determine for each voxel the probability that a random walker starting from there first reaches one of the user-specified foreground seeds. The probability with which a certain neighbor voxel is chosen as next step is defined by edge weights, which have to reflect the characteristics of the volume and are usually derived from intensity differences between the respective voxels. A crisp segmentation may finally be obtained by assigning each voxel to the label for which the highest probability was calculated.

Volume segmentation systems. In addition to the techniques outlined above, the research community has presented several volume segmentation systems, which can be classified based on accuracy, re- peatability and interaction efficiency [26]. Bartz et al. [1] have proposed a hybrid approach for the segmentation of the tracheo-bronchial tree of the lungs, where they limit the user interaction to the specification of seed points. Gu and Peters [14] have introduced a system for interactive organ segmentation based on the combination of an erosion operation and the fast marching method. Boykov and Jolly [3] employed the graph cuts technique for interactive organ segmentation. Ben-Zadok et al. [2] recently presented a volume segmentation tool based on level sets that focuses on image-guided therapy. Tzeng et al. [38] have presented a system which is based on a combination of machine learning and interactive painting. By interactively painting on slices, the system can be trained and the whole volume can be classified.

The systems named so far did not take into account the issue of classification uncertainty. In fact, though a variety of fully-automatic systems incorporating or providing probabilistic classification information exists, guided uncertainty-aware volume segmentation systems are rarely found. Cremers et al. [8] propose a probabilistic level set formulation for allowing the integration of user input into the segmentation process. Kontos et al. [22] presented a system for segmentation of medical data sets with respect to uncertainty. By combining manual thresholding and automated boundary correction, they are able to achieve accurate segmentations for 3D SPECT data. In contrast to our approach, uncertainty is not conveyed to the user, but only used for automatic improvements. More recently, Saad et al. [32] proposed an exploration and editing system for probabilistic segmentations of medical data sets. They analyze a given probabilistic segmentation in order to detect ambiguous regions and to allow the user to correct potential misclassifications. Though our approach might appear similar to theirs, there is a fundamental difference: instead of subsequently editing a given segmentation, our system focuses on the minimization of uncertainty already during the segmentation process. We consider this proceeding as preferable, since in our approach modifications to the probabilistic segmentation are directly based on the underlying volume data. While the basic interaction metaphor for defining seed points in our system is similar to the Volume Catcher system presented by Owada et al. [27], they do not consider and convey any uncertainty information.

Uncertainty visualization. Since uncertainty visualization plays a key role within our system, we briefly review the most relevant approaches. In their work originally targeted towards uncertainty visualization in a geospatial context, MacEachren et al. [24] classify uncertainty visualization based on data types and data quality. Although due to the context no volumetric uncertainty visualization is discussed, the principle of crispness and transparency can be transferred to our approach. In the same context, Pang [28] describes illustrative techniques for multivariate uncertainty data. Grigoryan and Rheingans [13] exploit uncertainty-based surface displacement. In their paper they also demonstrate the application to segmentation results. However, it requires a surface with an appropriate normal for which uncertainty information is present, which we cannot directly derive from our uncertain segmentation results. Pang et al. [29] describe alternative concepts for the visualization of surface uncertainty by means of bumps, glyphs and so-called fat surfaces. Even more relevant to our approach are the color, opacity and texture mapping techniques proposed by Rhodes et al. [31]. While this technique is also tailored towards surface representations, the speckle, texture and noise approach presented by Djurcilow et al. [9] has been developed for volumetric data sets. The same is true for the probabilistic animation approach presented by Lundström et al. [23].

\section{System Design}

In order to be able to provide an efficient guided segmentation workflow, the selection of an appropriate probabilistic segmentation algorithm was crucial for our system. In particular, we surveyed existing semi-automatic approaches with respect to the following requirements:

1. Availability of information about local reliability of an obtained segmentation. 


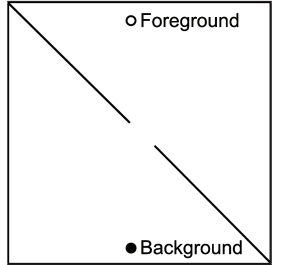

(a) Image with seeds

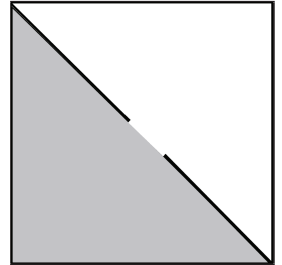

(b) Segmentation

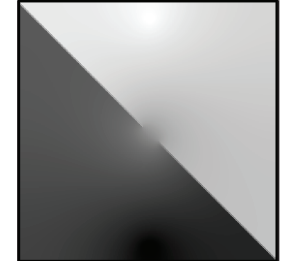

(c) Probability map
Fig. 2. Weak border detection property of the random walker: in contrast to the graph cut approach, the random walker is able to segment the objects although an interrupted boundary is present (adapted from [11]).

2. Efficient and reliable user interaction, i.e., small changes of the user-provided information should not be prone to cause large, unanticipated changes in the resulting segmentation.

\section{Fast computation.}

The first requirement already excludes the majority of techniques, including region-growing [16] and intelligent scissors [25], which provide binary segmentations. While there exist some fuzzy formulations of the level-set concept, which might allow one to derive uncertainty information, few of them have actually been applied to volumetric data, and to our knowledge all of these approaches are tailored towards specific use-cases, mainly brain MRI [6]. Additionally, due to the limited amount of interactivity, especially regarding the correction of intermediate results, level-sets do not seem to be well-suited for a guided workflow. While Ben-Zadok et al. [2] and Cremers et al. [8] presented promising advances towards interactive level set segmentation, their approaches do not provide probabilistic results.

Although the graph cuts technique itself is also limited to provide a crisp segmentation, Kohli and Torr [21] have shown recently how a confidence measure can be derived from a graph cut solution based on min-marginals. So we had the choice between graph cuts and the random walker approach, which is inherently probabilistic, since it provides each voxel's probability for the membership of all segments. The user interaction is very similar with both techniques: the user specifies two (small) sets of foreground and background seed voxels to sketch the desired segments. Thus, when dealing with wellshaped data sets, both algorithms are adequate. However, the "small cut" phenomenon of the graph cut algorithm hampers the reliability of the user interaction as illustrated in Figure 2: since the surface area of the provided seeds is smaller than the weak (missing) part of the boundary, the smallest cut surrounds the seeds assigning all pixels to the background (a), which is most likely not the result the user expected. Additionally, adding a rather small set of further seeds, so that the seed surface becomes larger than the weak boundary, would cause a drastic change in the segmentation towards the "correct" result. For $3 \mathrm{D}$ segmentation, the requirement to draw seed regions whose surface area exceeds the weak boundary area becomes even more problematic, since the user typically defines less seeds in comparison to the number of voxels than for 2D image segmentation. Figure 2 (b), in contrast, demonstrates the weak border detection property of the random walker, which is accompanied by the reflection of the weak boundary in the surrounding probabilities as shown in Figure 2 (c). Another problem of the graph cuts approach is its tendency to produce blocky artifacts at the object boundary [5], which especially becomes a problem when voxel-precise segmentation is desired.

For these reasons, we have selected the random walker algorithm to be the foundation of the proposed system. However, this decision is to some extent contrary to the requirement for a fast computation, since the graph cuts approach is known to run in real-time even for 3D data, whereas the time necessary for the computation of a random walker solution of medical data sets typically ranges from seconds to several minutes. To overcome this limitation, we exploit the features of current GPUs and provide an OpenCL implementation of a conjugate gradient solver used for the random walker calculations as described

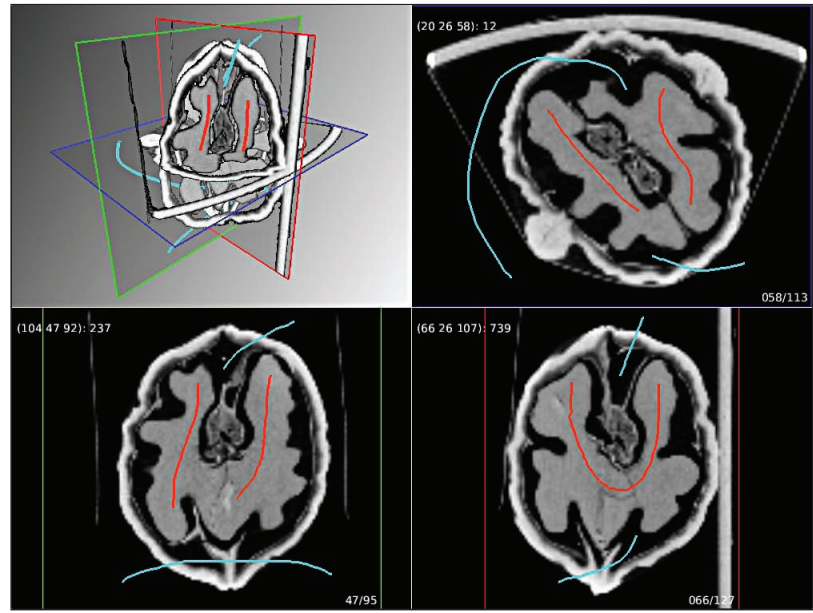

Fig. 3. User interface of the proposed system: The upper right, lower right and lower left views present axis-aligned slices of the data set to be segmented. The upper left multiplanar view conveys contextual information about the current slice positions. The user has drawn foreground seeds (red) and background seeds (blue) onto the 2D views.

in Section 6. Thus, we achieve computation times which support the interactive and iterative proceeding of our workflow.

\section{Guided Volume Segmentation}

Although uncertainty information is present in the probabilistic segmentation generated by the random walker algorithm, exploiting this information in a user-centric workflow is not trivial. In case of a multilabel segmentation, i. e., more than two segments are considered, the probabilities constitute a vector field, and even for two-label segmentation a combined $3 \mathrm{D}$ visualization of the data set and the probability volume seems to be hardly practical. In contrast, a manual slice-wise examination of probabilities and volume data is a cumbersome process for large data sets, posing the risk of missing critical misclassifications. The low-frequency character of the probability field further amplifies the risk of overlooking areas of uncertainty.

Throughout this paper, we adopt the commonly used medical data model, which assumes that a volume is composed of rather homogenous regions representing the scanned materials, which intermix at their borders constituting smooth transitions [19]. Under this assumption, the probabilistic segmentation can be expected to exhibit thin uncertainty margins at the boundaries. Therefore, large areas of ambiguous probabilities can be interpreted as indicators for potential misclassifications. We exploit this observation in order to derive information about the uncertainty of the segmentation result from the probability distribution.

The proposed system supports the user during the iterative refinement of probabilistic segmentations by analyzing the probability field to detect pronounced ambiguous regions and directing the user's attention to these. Since reliability is the main focus of our work, we have decided to employ a slice-based interface for user input (see Figure 3). The three axis-aligned slices are used for seed point definition as well as for displaying the current segmentation result along with ambiguous regions. The 3D multiplanar view provides context information about the current position of the slices in the data set and shows an iso-surface rendering of the segmentation with highlighted uncertain regions. Thus, we simultaneously convey the shape of the segmentation as well as the spatial relations of uncertain areas, which are both aspects that are difficult to perceive from a pure slice-wise representation. Although the random walker algorithm is applicable to K-way segmentation with an arbitrary number of labels, we decided to confine our system to 2-way segmentation, since the extraction of a single feature of interest from the background is the most common application for medical image segmentation and the simultaneous classification of multiple features would inevitably increase the complexity of 
the user interface. If necessary, multiple features can still be extracted iteratively.

The proposed segmentation system differs from previous work dealing with uncertainty in volume data in a significant way: Instead of just conveying the uncertainty of a given segmentation to the user or allowing the subsequent modification of the volume, as for instance proposed by Kniss et al. in the form of a Bayesian risk minimization framework [20], we consider uncertainty already in the segmentation stage and propose a combination of interaction and visualization techniques developed for allowing the user to reliably and efficiently minimize the uncertainty. When conducting first tests with the random walker technique, we noticed that for many use cases, as for instance organ segmentation, usually large parts of the desired object could be properly segmented quite easily, while ambiguous parts of the segmentation where rather small and scattered across the surface of the object, where they were sometimes hard to detect. Therefore, we based the design of our system on Shneiderman's visual information seeking mantra [37]: "Overview first, zoom and filter, then detailson-demand". Therefore, we not only exploit the system for actual computation of the random walker, but also for a preclassification of uncertain regions and the visual presentation of these. Thus the 'zoom and filter' process can be understood as a collaborative effort between user and system. By adapting this concept, we end up with an iterative segmentation process which reflects the described interplay between user and system. Thus, the detailed steps of the workflow shown in Figure 1 are:

1. The system generates an intermediate random walker segmentation using seed points defined in the previous iterations.

2. The system analyzes the probability field in order to detect ambiguous regions.

3. To provide an overview, all detected regions are displayed in a table and are highlighted in the 3D multiplanar view as well as on the slice views.

4. The following steps are repeated until the user decides to re-run the random walker with modified input parameters (seeds):

- The user focuses on a particular region by selecting it in the table widget: The multiplanar view and the slice views are adjusted to bring the selected region into focus.

- For further inspection the user may decide to zoom onto a selected region and locally refine the segmentation.

\subsection{Random Walker Parametrization}

Since the first step of our workflow relies on a proper parametrization of the random walker algorithm, some background information is required to fully understand our design considerations. The random walker algorithm as formulated by Grady [11] operates on a weighted graph, whose edge weights define the probability that the random walker chooses a certain neighbor node of its current location in the next step. For representing the structure of the volume to be segmented, we follow Grady's formulation of mapping each voxel to a node with edges representing its 6-neighborhood. To define the weight $w_{i j}$ of the edge connecting the nodes $i$ and $j$, a Gaussian function is used based on the difference between the intensities $g_{i}$ and $g_{j}$ of the corresponding voxels:

$$
w_{i j}=\exp \left(-\beta\left(g_{i}-g_{j}\right)^{2}\right)
$$

The only free parameter $\beta$ can be interpreted as the permeability of boundaries: increasing $\beta$ causes a larger decrease of edge weights within areas of high gradient magnitude than within more homogeneous regions. However, according to our experience, this parameter is rather insensitive: A value of around 4000 gave reliable results for data sets of different sizes and modalities. In our experiments, solely the segmentation of very thin structures, which is an application the random walker algorithm is less suited for, required an adjustment of $\beta$ to values between 8000 and 16000 . While the output of the random walker are probability values, the user also inputs probabilities represented by seed nodes. This is done by assigning probability values of 0.0 and 1.0 to background and foreground seeds. Based on this input, the random walker solution assigns to each unseeded node $n_{i}$ the probability with which a random walker starting from $n_{i}$ reaches one of the foreground seeds. Due to this reason, the probabilities are also commonly regarded as foreground membership scores.

For incorporating an arbitrary transfer function in the segmentation process, we modify the edge weight $w_{i j}$ by adding a term representing the distance between the optical properties assigned to the voxels corresponding to nodes $i$ and $j$. In the following definition, $\vec{f}(i)$ denotes the color that is assigned to node $i$, abstracting from the actual domain of a specific transfer function:

$$
w_{i j}=(1-\alpha) \exp \left(-\beta\left(g_{i}-g_{j}\right)^{2}\right)+\alpha \exp \left(-\beta(\vec{f}(i)-\vec{f}(j))^{2}\right)
$$

We limit the weighting factor $\alpha$ to the range $[0,0.5]$, in order to ensure that the obtained segmentation is always based on the original data and not solely derived from the user-provided classification.

\subsection{Uncertainty Detection}

The ability to reliably detect uncertainty in the probabilistic segmentation result is crucial for our system. First of all, since even to obvious background or foreground parts no clear 0.0 or 1.0 probabilities are assigned, we perform a thresholding in order to suppress these obvious parts and thus extract the suspicious areas. The used thresholds can be set interactively; however, based on our experience with CT and MRI data, we have identified 0.2 to 0.8 as the probability range containing all uncertain regions. All probabilities lying outside this range can be classified as certain. Choosing a large threshold range would be insufficient, since it would enclose certain regions, e.g., 0.1 is often assigned to obvious background parts that are not close to a seed.

The thus thresholded probability distribution usually still contains large parts of clearly classified boundaries. This is caused by the fact that due to the partial volume effect boundaries in scanned data sets do not appear as sharp edges but as rather smooth transitions. Therefore, instead of a hard drop-off we usually find a probability profile resembling the boundary intensity profile in the data set. Applying an erosion operation to the filtered probability field could in principle remove these boundaries, however at the risk of accidentally pruning smaller uncertain structures. Instead, we apply a gradient length threshold to the probability field, which we derive from the user-specified maximum expected width $d_{b}$ of certain boundaries: assuming a linear probability profile across the boundary, the maximal tolerable gradient magnitude is defined as $(0.8-0.2) / d_{b}$. Such an edge filter works much more reliably on the probability field than in the data domain. This is due to the inherently smooth nature of the probability field, which is given, since each unseeded voxel represents the weighted average of its neighbors' probabilities [11]. After this boundary suppression has been performed, we obtain the relevant areas of uncertainty. To be able to effectively guide the user through the distribution of uncertainty, we perform a connected component analysis yielding the coherent regions of uncertainty finally presented to the user.

To further support the user when processing the identified uncertain regions, we display them in the table widget sorted by their importance. To estimate this importance of an uncertain region $R$, we introduce a measure for ambiguity $\operatorname{amb}(R)$, that takes into account both the size of the region and its probability distribution $p$ :

$$
\operatorname{amb}(R):=\sum_{v \in R}(1-2 \cdot|p(v)-0.5|)
$$

The term $1-2 \cdot|p(v)-0.5|$ specifies the uncertainty of a voxel $v$, since it is inversely proportional to the distance of $p(v)$ to the most ambiguous probability value 0.5 . Thus, the user can process the uncertain regions based on this order as displayed in the table widget.

\subsection{Refining the Segmentation}

In the usual case, ambiguity is caused by an insufficient number of seed points in the neighborhood. This is characterized by an uncertain 


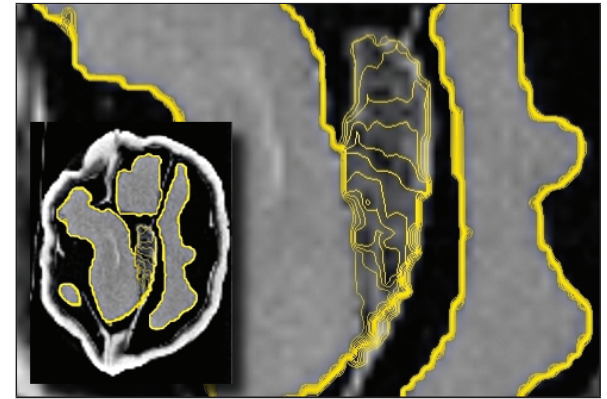

(a)

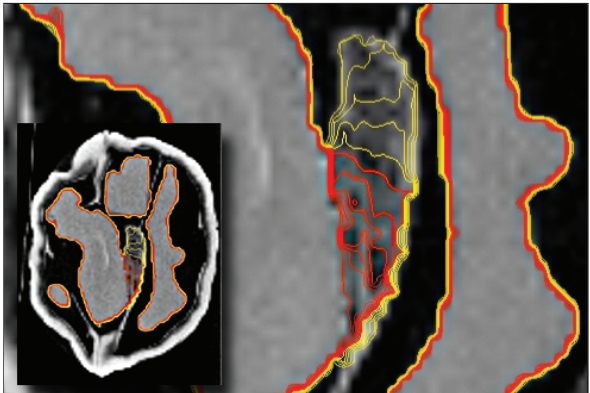

(b)

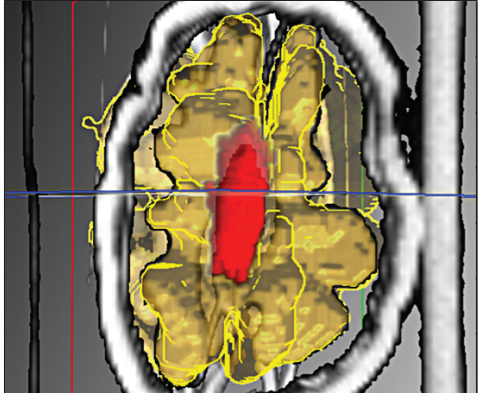

(c)

Fig. 4. To convey the uncertainty of the segmentation results, we employ different uncertainty visualization techniques. In 2D, we propose the use of uncertainty isolines which depict the uncertainty inherently through the inter-line distance (a). These isolines can also be subject to a two-color mapping (b). In 3D, where an overview depiction is demanded, we use opacity modulation together with contour emphasized surfaces (c).

area crossing a boundary, which in principle should provide sufficient contrast for a clear classification. This type of ambiguity can be efficiently solved by adding a few more seeds for both the fore- and background segment. Alternatively, when adding seed points one by another is not sufficient, the user may define all seed points on the edge directly by using a geometry widget. One way to perform this is to use a curve editor, which supports the user when fitting a curve to the boundary. Based on this curve multiple seed points are automatically set.

\section{UNCERTAINTY VISUALIZATION}

To better guide the user's attention to the detected regions having a high uncertainty, we have tested different uncertainty visualization techniques. Thus, we are able to provide a meaningful emphasis of the detected uncertain regions, while also conveying the overall quality of the final result. Based on the multiple linked view setup of our system, we depict uncertainty in both the $2 \mathrm{D}$ as well as the $3 \mathrm{D}$ view. While $2 \mathrm{D}$ slice views are well-suited for precise inspections, 3D visualizations have the advantage that they provide an expressive overview of structures. To achieve meaningful uncertainty visualizations, we take these qualities into account. Thus, the 2D uncertainty visualization should be able to depict all relevant details regarding the uncertainty, while the $3 \mathrm{D}$ view should provide a meaningful overview of the uncertain regions.

2D techniques. To depict the uncertainty in the 2D slice views, we propose the usage of uncertainty isolines, as shown in Figure 4 (a). These isolines are directly derived by applying image processing operators to the probability distribution of the current slice. To reduce cluttering of the original data augmented by the isolines, we first mask regions with probability values outside the defined uncertainty range between 0.2 and 0.8 , then apply a quantization operator, and finally perform an edge detection on the quantized probability field. Thus, all regions with uncertain probability values are covered by isolines, while isolines at well-classified boundaries collapse to a sharp contour, since these boundaries are characterized by a strong drop of probability values. When choosing the number of quantization steps, we have to make a trade-off between occlusion resulting from a too dense set of isolines, and loss of focus due to too few isolines. According to our experience, a number of 10 till 20 isolines seems to be appropriate.

Besides the actual uncertainty visualization strategy, the color mapping used to depict the uncertainty also has to be chosen carefully. In our initial implementation, we had integrated a two-color mapping based on two distinct hues (see Figure 4 (b)). While lightness variations of the one hue were used for all probability values below 0.5 , shades of the other hue were used for all probability values above 0.5 . However, since changes in hue are perceived pre-attentively [39], this led to an inherent emphasis of one potential boundary, along the 0.5 uncertainty isoline. Since there is no evidence that this is the most probable boundary, we have omitted this two-color mapping as shown in Figure 4 (a).
3D techniques. While already a substantial amount of research has been conducted to improve uncertainty visualization in $3 \mathrm{D}$, the existing techniques are only applicable up to a certain extent in our case. Especially those techniques dedicated to visualizing surface uncertainty $[29,13]$ were not very well suited, since we wanted to avoid introducing the notion of a surface in uncertain regions. Instead, in order to emphasize the volumetric nature of the uncertain region, uncertain volume visualization techniques are more adequate. We exploit a volumetric uncertainty visualization, which is inspired by the transparency approach described by MacEachren et al. [24]. Therefore, we apply an opacity transfer function to the uncertain regions, which sets the hue to constant and modulates the transparency based on the probability values. This leads to a blobby appearance, which fades out towards the areas of higher uncertainty. In Figure 4 (c) we show the outcome of this technique in combination with a surface representation of the certain segment boundaries and a multiplanar view providing contextual information. As it can be seen, transparency is exploited when visualizing the segmentation boundary in order to deal with the occlusion problem. However, since transparency affects the shape perception of this boundary [17], we have augmented the visualization by adding contour edges, which we have derived based on a Sobel filter applied to the corresponding depth values. The positive effects of such contour enhancement have also been described by other authors, when dealing with semi-transparent surfaces [10]. While the transparency modulation also influences shape perception, this is not an issue in our case, since the 3D view is only intended to provide an overview, while the $2 \mathrm{D}$ view is used to explore structures in more detail.

\section{IMPLEMENTATION}

The visualization components of the proposed system have been implemented using OpenGL and GLSL achieving interactive performance. The basic filtering operations as well as the connected component analysis performed on the probability field last less than $0.5 \mathrm{~s}$ for volumes of size $256^{3}$. Therefore, the main challenge for an interactive workflow was to realize an efficient implementation of the random walker method. Grady [11] exploited the equivalence between the random walker problem and the Dirichlet problem from potential theory to transform the computation of the random walker probabilities into the solution of a system of linear equations. Though this system is large, containing one equation per unseeded voxel, the corresponding matrix is also sparse and positive definite, allowing the application of efficient iterative solvers like conjugate gradients. However, interactive speeds on the CPU were only achieved for medium-sized 2D images in [11], which matched our experiences with CPU-based conjugate gradient solvers. The OpenGL-based GPU implementation of the Random Walker presented in [12], on the contrary, enabled interactive results for $3 \mathrm{D}$ volumes of sizes up to $128^{3}$.

Therefore, it was obvious that we had to exploit the processing power of the GPU for allowing an interactive workflow. We favored 
an OpenCL-based implementation of the conjugate gradient method over an GLSL-based approach due to the straightforward programming model, for instance not requiring the encoding of data into textures. Since the sparse matrix representing the random walker equation system can be reduced to three elements per row [12] and the conjugate gradient method requires three additional vectors for temporary storage, six values need to be stored in the GPU memory for each unseeded voxel. Therefore, our single-precision ( 4 byte floats) implementation requires 24 bytes per unseeded voxel to be stored. On our system equipped with an NVIDIA GeForce GTX 285 graphics board with 1 GB graphics memory we were able to process volumes with approximate maximal dimensions of $300^{3}$, which corresponds to a memory consumption of about $633 \mathrm{MB}$. Presumably, the remaining graphics memory is occupied by OpenGL resources, such as volume textures. By storing the matrix with half-precision, however, we were able to elevate this limit to approximately $350^{3}$, with neglectable impact on the runtime. When initializing our conjugate gradient solver with the result of the previous iteration of the workflow, one computation of the random walker solution for a $256^{3}$ volume takes about 4-6 seconds. The first iteration of the system after the initial seed placement usually requires $20-30$ seconds.

\section{REsULtS}

To judge the benefits of the proposed system, we have performed an informal as well as a formal user study. Furthermore, we have quantitatively analyzed the obtained segmentation results, which we have compared to ground truth segmentations.

\subsection{User Study}

The informal user study was two-fold. In the first test, we have asked three users to segment a data set by using our system and a comparison system which is based on region growing. The second part of the study has been conducted as an informal interview with a medical expert.

Among the three users participating in the informal study where two male and one female, one of these persons had previous experience with volume segmentation techniques. Each of these users had to segment the seed of the walnut data set shown in Figure 3. This data set has been chosen, since besides several interior regions, which can be easily segmented, also several ambiguous regions occur, which suffer from a high degree of uncertainty. For some of these regions it is even difficult for a human observer to spot the most likely boundary. We have asked two of the users to first segment the walnut's interior by using our system and afterwards by using the region growing approach. The third user had to perform the segmentation tasks in the reverse order. For both approaches we gave a very short introduction, which took less than a minute. All users needed less than 5 iterations of the workflow when segmenting the interior with the proposed system, which took them between 3-5 minutes. After the tests have been completed, all users stated that our underlying workflow has been per-

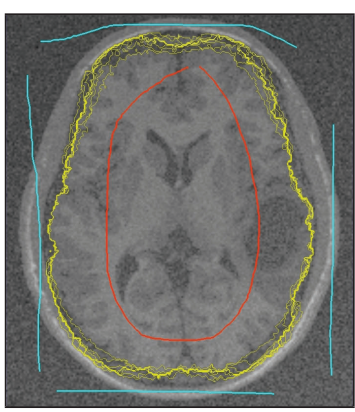

(a)

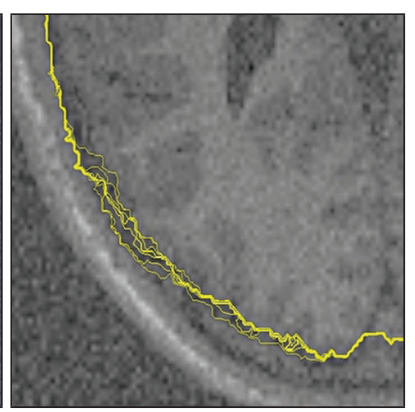

(b)
Fig. 5. Typical workflow of the conducted user study. (a) shows the initial seed placement on an axial slice of the MRI scan along with a large uncertain layer enclosing the brain, while in (b) the system has zoomed onto a smaller uncertain region during a subsequent iteration.

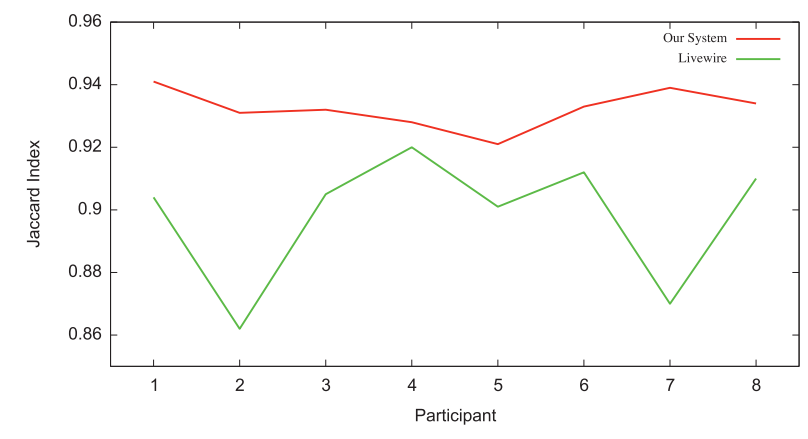

Fig. 6. Quantitative evaluation of the user study showing Jaccard indices between the user-created segmentations and the ground truth.

ceived as very helpful. Especially the user who had prior experience with volume segmentation appreciated the ease of use as well as the quality of the result. While the users needed roughly the same time to perform the task with the region-growing technique, they could not achieve comparable results. Furthermore, it was not clear to the users when a sufficient quality of the result was achieved, and thus their confidence in the segmentation quality was lower.

The informal interview with the medical expert led to comparable results. Similar to the user, who had previous segmentation experience, the medical expert especially emphasized the importance of the workflow. Additionally, the conveying of the reliability of the segmentation was appreciated. Based on the interview and the shown demonstration, we could arouse interest, and it is planned that the system is used for medical research in the future.

Based on this initial feedback we have then conducted a formal user study involving quantitative analysis of the segmentation results achieved by the participants. As a practical application case we selected a data set from the TumorSim database [30], which provides simulated brain tumor MRI scans along with ground truth segmentations. Since these data sets are designed for a realistic validation of brain segmentation algorithms, they suffer from similar deficiencies as real brain MRI scans, especially high noise and bias (see Figure 5). The participants were asked to segment the complete brain including the tumor, first with our proposed system and afterwards with a livewire approach. Out of the available MRI modalities we have chosen the T1 data set (256x256x175), in which the brain exhibits the least contrast to the surrounding tissue and which therefore appears to be the most challenging segmentation task. After a short introduction to both systems the two female and six male participants were given at most $15 \mathrm{~min}$ for segmenting the brain with each of the systems. With the random walker approach only one user took advantage of the full time limit, while the remaining participants needed between 8 and 13 minutes. During the initial seed placement phase, most users marked the brain as foreground and the skull as background, but did not place any seeds in the gap between brain and skull. Therefore, after the first iteration, the system typically detected a large layer enclosing the brain as uncertain, as shown in Figure 5(a). After additional background seeds had been placed in this gap, it usually decayed into several smaller uncertain regions, which were then worked through by the users during subsequent iterations. Within the livewire system, the user has to mark the contour of the region to be segmented slice-by-slice. It supports the user by offering contour snapping based on intensity differences and it also provides interpolation of contours across slices. Due to the limited amount of time, the participants were able to process at most 30 out of the approx. 150 slices actually containing brain tissue and had therefore to interpolate contours for the intermediate, unprocessed slices. Four participants took the full 15 minutes, the remaining ones needed more than 10 minutes.

When requested to rate the correctness of the segmentations generated with both approaches, most participants showed slightly higher confidence in the correctness of their random walker segmentation. To be able to judge the accuracy of the segmentation, we have further 
performed a quantitative analysis. Therefore, we have computed the Jaccard index [35] between the user-generated segmentations and the ground truth. The Jaccard index measures the similarity between two sample sets $A$ and $B$ and is defined as the size of their intersection divided by the size of their union:

$$
J(A, B):=\frac{|A \cap B|}{|A \cup B|} \quad \in[0 ; 1]
$$

As shown in Figure 6, all participants achieved significantly more accurate results with the proposed system than with the livewire technique. Furthermore, all users were able to achieve a rather accurate segmentation with our approach, while higher variance occurred when comparing the livewire segmentations.

To evaluate the effectiveness of the different system components, we have asked the users to estimate the risk of overlooking critical segmentation errors. Therefore, they had to rate this risk in a postquestionnaire, under four different assumptions: first, when using the proposed system; second, when only using the user guidance; third, when only using the uncertainty visualization; and fourth, when using no user guidance and no uncertainty visualization. On a scale from 0 to 5, where 5 meant to miss critical errors for sure, all users have rated the proposed system with a score smaller or equal to 2 . In contrast, all participants have assessed the absence of one of the components with a score of 3 or higher, and four of them have rated the risk as 5 for a system lacking both components. Finally, the participants were asked to judge which system would allow them to obtain the most accurate segmentation with unlimited amount of time. While three participants rated both systems equally in that regard, the remaining users favored the proposed system over the livewire approach. Being asked for the reasons, most of them stated that though they consider it in principle possible to create a perfect segmentation with the livewire technique, the large amount of work required would inevitably cause a human to make mistakes. Two users were generally skeptical about their ability to precisely sketch complex contours and therefore favored the random walker system, which only required them to place seeds close to a boundary.

\subsection{Result Comparison}

We have applied our system to two application cases: Abdominal CT and brain MRI data. Figure 7 shows how the system has been used to segment a liver from an abdominal CT data set. As Figure 7 (a) shows, initially a large number of uncertain regions has been detected. After reducing the number of regions during approximately 5 minutes in 5 iterations, the results shown in Figure 7 (b) have been achieved.

The results in Figure 8 show the outcome when applying the system to the T2 modality of the TumorSim MRI data set. Due to the high contrast of the brain boundary in this modality, we were able to obtain

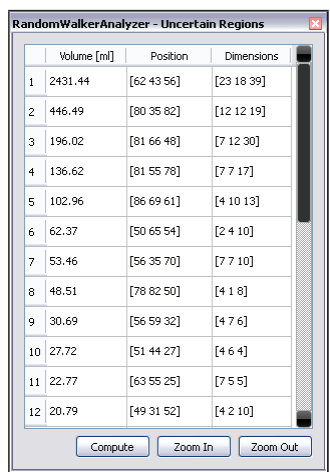

(a)

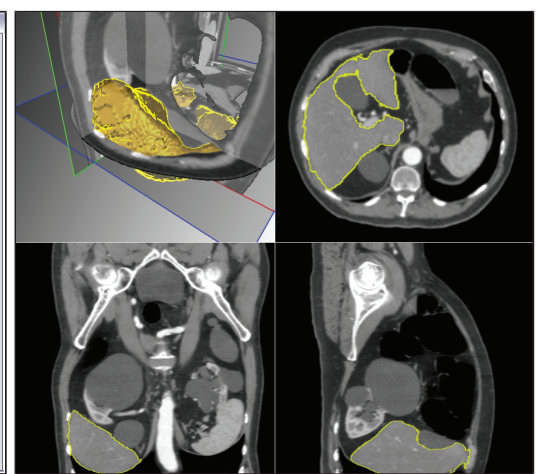

(b)
Fig. 7. The proposed system applied to abdominal CT data. The images show the representations generated when segmenting the liver: the table displaying the initial uncertain regions (a) and the final result as shown by the system (b).

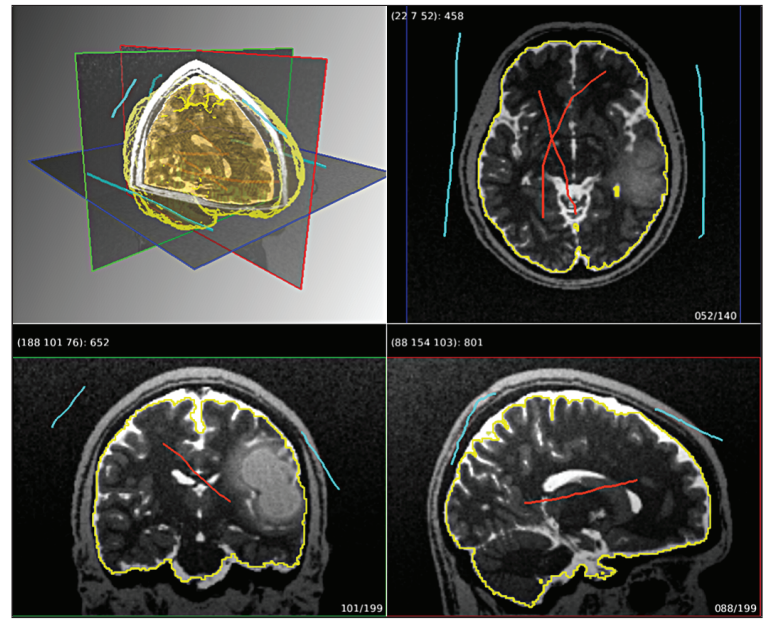

Fig. 8. The results of using the proposed system on simulated brain MRI T2 data from the TumorSim database [30] (Jaccard: 0.993).

a high-quality segmentation (Jaccard: 0.993) after only three iterations and 2 minutes. For a more challenging segmentation task, we applied our technique to the T1 modality (Case 2) of the brain MRI data from the 2010 Visualization Contest (see Figure 9), which also provides ground truth for the brain as well as the tumor. It took us 15 minutes to extract the brain (Jaccard: 0.953 ) and 5 minutes to segment the tumor (Jaccard: 0.937)

\subsection{Limitations}

A major drawback of the proposed system is the limited applicability of the random walker technique to thin structures. With the proposed technique, we were able to easily segment longitudinal structures with a certain thickness, such as the spinal cord in the MRI data sets or the aorta in contrast-enhanced CT. However, segmenting the thin brain vessels in the MRI scans was only possible by an excessive placement of seeds. Nevertheless, according to our experience this is an inherent limitation of many seed-based segmentation approaches.

\section{Conclusions and Future Work}

In this paper we have presented an interactive system based on the random walker technique which allows the user to generate reliable segmentations by incorporating uncertainty information. The system is based on a carefully designed iterative workflow, which exploits the strengths of a computer system and the human user by establishing a tightly coupled interaction between these two. Within the proposed workflow, uncertain regions of the segmentation are automatically detected and iteratively presented to the user for further refinement. In contrast to previous techniques, uncertainty visualization is interweaved within the whole workflow, and thus the user is always aware of the reliability of the segmentation achieved so far. To be able to communicate with the system, we support the user by allowing to draw rough strokes and to use other more precise interaction widgets. Based on the thus defined input, we directly parametrize the exploited random walker segmentation algorithm, which in comparison to other segmentation editing approaches has the advantage that we directly work on the original data, instead of modifying the resulting segmentation. Furthermore, we could show how to exploit the OpenCL programming API and thus the vast processing power of modern GPUs in order to realize a random walker implementation which is fast enough to support the proposed workflow. To evaluate the usefulness of the presented approaches, we have conducted user studies. The results of these tests indicate that the presented system allows fast and accurate segmentation by supporting user confidence regarding the results.

In the future we see several possibilities to conduct research for extending the presented system. A stronger adaption of the random walker technique itself to certain application cases might prove valu- 


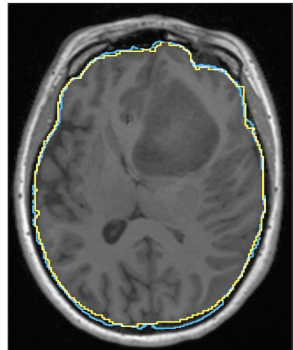

(a)

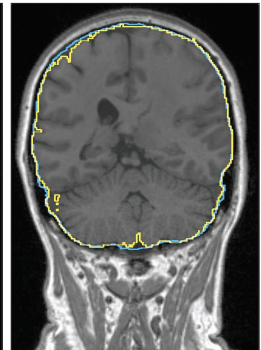

(b)

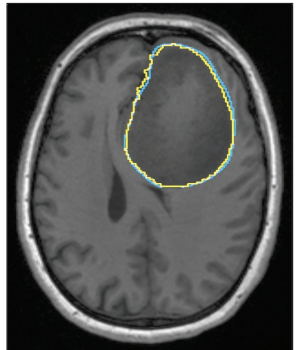

(c)
Fig. 9. Application of the proposed system to a T1 brain MRI scan from the 2010 Visualization Contest. (a) and (b) show axial and coronal slices, respectively, overlaid with the obtained brain segmentation (yellow) as well as the ground truth (blue). (c) displays the result of the tumor segmentation. Jaccard brain: 0.953, Jaccard tumor: 0.937.

able. For instance, it should be evaluated whether more advanced edge weight definitions could improve the applicability of the system to the segmentation of very thin structures. Furthermore, an in-depth analysis should be conducted in order to analyze which transfer function spaces can be optimally combined with the presented approach. In particular more sophisticated classifiers, such as for instance shapebased [33] or occlusion-based classifiers [7], should be investigated.

\section{ACKNOWLEDGEMENTS}

This work was partly supported by grants from the Deutsche Forschungsgemeinschaft (DFG), SFB 656 MoBil Münster, Germany (project Z1). The presented concepts have been integrated into the Voreen volume rendering engine (www.voreen.org). The 2010 Visualization Contest data set is courtesy of Prof. B. Terwey, Klinikum Mitte, Bremen, Germany.

\section{REFERENCES}

[1] D. Bartz, D. Mayer, J. Fischer, S. Ley, A. d. Rio, S. Thust, C. P. Heussel, H.-U. Kauczor, and W. Strasser. Hybrid segmentation and exploration of the human lungs. In IEEE Visualization, pages 177-184, 2003.

[2] N. Ben-Zadok, T. Riklin-Raviv, and N. Kiryati. Interactive level set segmentation for image-guided therapy. In IEEE Int. Symp. on Biomedical Imaging, pages 1079-1082, 2009.

[3] Y. Boykov and V. Kolmogorov. Interactive organ segmentation using graph cuts. In Int. Conf. on Medical Image Computing and ComputerAssisted Intervention, pages 276-286, 2000.

[4] Y. Boykov and V. Kolmogorov. Interactive graph cuts for optimal boundary and region segmentation of objects in n-d images. In IEEE Int. Conf. on Computer Vision, pages 105-112, 2001.

[5] Y. Boykov and V. Kolmogorov. Computing geodesics and minimal surfaces via graph cuts. In IEEE Int. Conf. on Computer Vision, pages 26-33, 2003.

[6] Z. Chen, T. Qiu, and S. Ruan. A brain tissue segmentation approach integrating fuzzy information into level set method. Automation and Logistics (ICAL), pages $1216-1221,2008$.

[7] C. D. Correa and K.-L. Ma. The occlusion spectrum for volume classification and visualization. IEEE TVCG, 15(6):1465-1472, 2009.

[8] D. Cremers, O. Fluck, M. Rousson, and S. Aharon. A probabilistic level set formulation for interactive organ segmentation. Medical Imaging 2007: Image Processing, 6512(1):120-129, 2007.

[9] S. Djurcilov, K. Kim, P. F. J. Lermusiaux, and A. Pang. Volume rendering data with uncertainty information. In Joint EUROGRAPHICS/IEEE TCVG Symp. on Visualization, pages 243-252, 2001.

[10] J. Fischer, D. Bartz, and W. Straßer. Illustrative Display of Hidden IsoSurface Structures. In IEEE Visualization, pages 663-670, 2005.

[11] L. Grady. Random walks for image segmentation. IEEE Trans. on Pattern Analysis and Machine Intelligence, 28(11):1768-1783, 2006.

[12] L. Grady, T. Schiwietz, S. Aharon, and T. U. München. Random walks for interactive organ segmentation in two and three dimensions: Implementation and validation. In MICCAI 2005 II, pages 773-780, 2005.

[13] G. Grigoryan and P. Rheingans. Point-based probabilistic surfaces to show surface uncertainty. IEEE TVCG, 10(5):564-573, 2004.
[14] L. Gu and T. Peters. Robust 3d organ segmentation using a fast hybrid algorithm. In Computer Assisted Radiology and Surgery, volume 1268, pages $69-74,2004$.

[15] Hastreiter, Peter and Ertl, Thomas. Fast and Interactive 3D-Segmentation of Medical Volume Data. In Computer Graphics International, pages $78-85,1998$.

[16] R. Huang and K.-L. Ma. Rgvis: Region growing based techniques for volume visualization. In Pacific Conf. on Computer Graphics and Applications, pages 355-363, 2003.

[17] V. Interrante, H. Fuchs, and S. M. Pizer. Conveying the $3 d$ shape of smoothly curving transparent surfaces via texture. IEEE TVCG, 3(2):98117, 1997.

[18] C. Johnson. Top scientific visualization research problems. IEEE Computer Graphics and Applications, 24(4):13-17, 2004.

[19] G. Kindlmann and J. W. Durkin. Semi-automatic generation of transfer functions for direct volume rendering. In IEEE Symp. on Volume Visualization, pages 79-86, 1998.

[20] J. M. Kniss, R. V. Uitert, A. Stephens, G.-S. Li, T. Tasdizen, and C. Hansen. Statistically quantitative volume visualization. In IEEE Visualization, page 37, 2005.

[21] P. Kohli and P. H. Torr. Measuring uncertainty in graph cut solutions. Computer Vision and Image Understanding, 112(1):30 - 38, 2008.

[22] D. Kontos, Q. Wang, V. Megalooikonomou, A. H. Maurer, L. C. Knight, S. Kantor, H. P. Simonian, and H. P. Parkman. A tool for handling uncertainty in segmenting regions of interest in medical images. Int. J. of Intelligent Systems Technologies and Applications, 1(3/4):194-210, 2006.

[23] C. Lundström, P. Ljung, A. Persson, and A. Ynnerman. Uncertainty visualization in medical volume rendering using probabilistic animation. IEEE TVCG, 13(6):1648-1655, 2007.

[24] A. M. Maceachren, A. Robinson, S. Gardner, R. Murray, M. Gahegan, and E. Hetzler. Visualizing geospatial information uncertainty: What we know and what we need to know. cartography and geographic. Information Science, 32:137-160, 2005.

[25] E. N. Mortensen and W. A. Barrett. Intelligent scissors for image composition. In SIGGRAPH '95, pages 191-198, 1995.

[26] S. Olabarriaga and A. Smeulders. Interaction in the segmentation of medical images: A survey. Medical Image Analysis, 5(2):127 - 142, 2001.

[27] S. Owada, F. Nielsen, and T. Igarashi. Volume Catcher. In Symp. on Interactive 3D Graphics and Games, pages 111-116, 2005.

[28] A. Pang. Visualizing uncertainty in geo-spatial data. In Work. Intersections between Geospatial Information and Information Technology, 2001.

[29] A. T. Pang, C. M. Wittenbrink, and S. K. Lodh. Approaches to uncertainty visualization. The Visual Computer, 13:370-390, 1996.

[30] M. Prastawa, E. Bullitt, and G. Gerig. Synthetic ground truth for validation of brain tumor mri segmentation. In MICCAI, pages 26-33, 2005.

[31] P. J. Rhodes, R. S. Laramee, R. D. Bergeron, and T. M. Sparr. Uncertainty visualization methods in isosurface volume rendering. In Eurographics 2003, pages 83-88, 2003.

[32] A. Saad, T. Möller, and G. Hamarneh. Probexplorer: Uncertainty-guided exploration and editing of probabilistic medical image segmentation. Joint Eurographics/IEEE-VGTC Symp. on Visualization, 29(3), 2010.

[33] Y. Sato, C.-F. Westin, A. Bhalerao, S. Nakajima, N. Shiraga, S. Tamura, and R. Kikinis. Tissue classification based on $3 \mathrm{~d}$ local intensity structures for volume rendering. IEEE TVCG, 6(2):160-180, 2000.

[34] J. A. Sethian. Level Set Methods and Fast Marching Methods: Evolving Interfaces in Computational Geometry, Fluid Mechanics, Computer Vision, and Materials. Cambridge University Press, 2 edition, June 1999.

[35] D. W. Shattuck, G. Prasad, M. Mirza, K. L. Narr, and A. W. Toga. Online resource for validation of brain segmentation methods. NeuroImage, 45(2):431 - 439, 2009.

[36] A. Sherbondy, M. Houston, and S. Napel. Fast volume segmentation with simultaneous visualization using programmable graphics hardware. In IEEE Visualization, pages 171-176, 2003.

[37] B. Shneiderman. The eyes have it: A task by data type taxonomy for information visualizations. In IEEE Vis. Languages, pages 336-343, 1996

[38] F.-Y. Tzeng, E. B. Lum, and K.-L. Ma. An intelligent system approach to higher-dimensional classification of volume data. IEEE TVCG, 11(3):273-284, 2005

[39] C. Ware. Information Visualization: Perception for Design. Morgan Kaufmann Publishers Inc., 2000.

[40] S. C. Zhu and A. Yuille. Region competition: Unifying snakes, region growing, and bayes/mdl for multiband image segmentation. IEEE Trans. on Pattern Analysis and Machine Intelligence, 18:884-900, 1996. 